OPEN ACCESS

Edited by:

Elisabetta Ada Cavalcanti-Adam, Max-Planck-Gesellschaft, Germany

Reviewed by:

Adil Denizli,

Hacettepe University, Turkey Andre D. R. Sliva,

Brazilian Air Force Academy, Brazi

${ }^{*}$ Correspondence:

Yoshihiro Yui

yoshihiro.yui@tokushukai.jp

Specialty section:

This article was submitted to

Biomaterials,

a section of the journa

Frontiers in Materials

Received: 03 December 2020 Accepted: 12 January 2021

Published: 24 February 2021

Citation:

Kumai J, Sasagawa S, Horie M and

Yui Y (2021) A Novel Method for

Polyacrylamide Gel Preparation Using

$N$-hydroxysuccinimide-acrylamide

Ester to Study Cell-Extracellular Matrix

Mechanical Interactions.

Front. Mater. 8:637278

doi: 10.3389/fmats.2021.637278

\section{A Novel Method for Polyacrylamide Gel Preparation Using N-hydroxysuccinimide-acrylamide Ester to Study Cell-Extracellular Matrix Mechanical Interactions}

\author{
Jun Kumai ${ }^{1}$, Satoru Sasagawa ${ }^{1}$, Masanobu Horie ${ }^{2}$ and Yoshihiro Yui ${ }^{1 *}$ \\ ${ }^{1}$ Research Institute, Nozaki Tokushukai Hospital, Osaka, Japan, ${ }^{2}$ Radioisotope Research Center, Division of Biochemical \\ Engineering, Kyoto University, Kyoto, Japan
}

Mechanical stimulation by the extracellular matrix (ECM) controls physiological and pathological cellular responses, such as stem cell differentiation, organogenesis, and tumor progression. Polyacrylamide (PA) gels have been widely used to study cell-ECM mechanical interactions. Typically, sulfosuccinimidyl 6-(4'-azido-2'-nitrophenylamino) hexanoate (sulfo-SANPAH) is used as a protein crosslinker in these gels. However, its low solubility, unstable binding with proteins, and high cost are barriers to its application. The objective of this study was to improve and simplify the preparation of PA gels using an economical crosslinker, N-hydroxysuccinimide-acrylamide (NHS-AA) ester, to enable increased stability in protein coating. By exposing excess NHS to the gel surface, we found an optimal ratio of NHS-AA ester:AA to obtain NHS-AA ester-containing PA gels with a uniform ECM protein coating and stiffness similar to that of sulfo-SANPAHcontaining PA gels. The biological behavior of MCF7 and MCF10A cells were similar on NHS-AA ester and sulfo-SANPAH gels. Acini formation in Matrigel overlay culture were also consistent on NHS-AA ester and sulfo-SANPAH gels. This novel PA gel preparation method using NHS-AA ester can effectively replace the sulfo-SANPAH method and will be immensely useful in the evaluation of cell-ECM mechanical interactions.

Keywords: polyacrylamide gel, N-hydroxysuccinimide-acrylamide ester, extracellular matrix, sulfosuccinimidyl 6(4'-azido-2'-nitrophenylamino)hexanoate, mechanical stimulation

\footnotetext{
Abbreviations: ECM, extracellular matrix; PA, polyacrylamide; AA, acrylamide; sulfo-SANPAH, sulfosuccinimidyl 6-(4'azido-2'-nitrophenylamino)hexanoate; NHS-AA: N-hydroxysuccinimide-acrylamide, PEG, polyethylene glycol; UV, ultraviolet; ACA, N-acryloyl-6-aminocaproic acid; N6, N-succinimidyl ester of acrylamidohexanoic acid; EGF, epidermal growth factor; AFM, atomic force microscopy; EGFP, enhanced green fluorescent protein; $\mathrm{p}$-FAK, phosphorylated focal adhesion kinase; YAP, Yes1 associated transcriptional regulator; DMEM, Dulbecco's modified Eagle's medium; APTES, 3-aminopropyltriethoxysilane; DCDMS, dichlorodimethylsilane; DW, deionized water; RT, room temperature; PBS, phosphatebuffered saline; BSA, bovine serum albumin; PCR, polymerase chain reaction; SD, standard deviation; 3D, three-dimensional; MCF7 and MCF10A, Michigan Cancer Foundation 7 and 10A, respectively.
} 

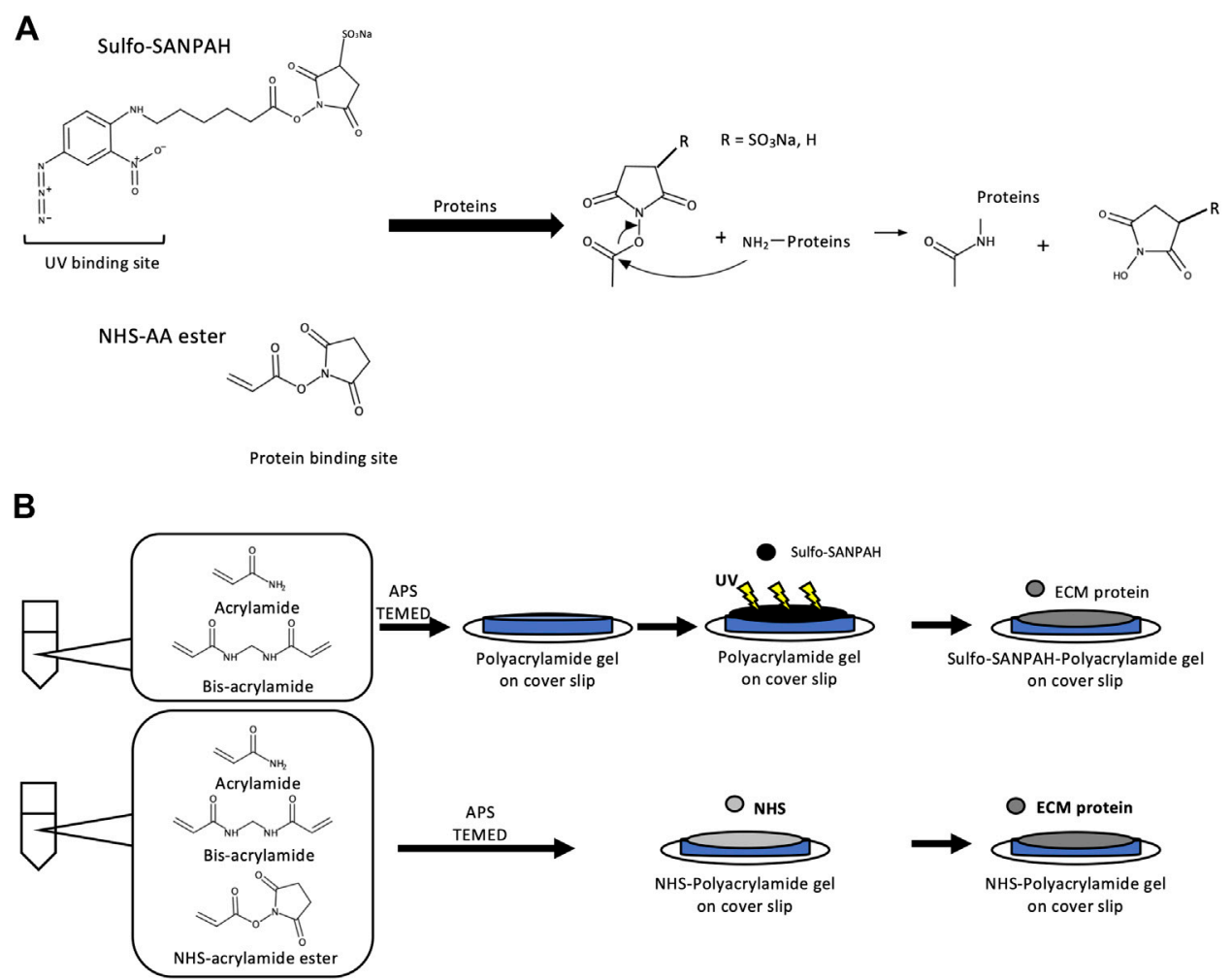

FIGURE 1 | Overview of protein crosslinker action and PA gel preparation (A) When sulfo-SANPAH is used as a protein crosslinker, the ECM protein forms an amide bond with the reactive site of sulfo-SANPAH and indirectly binds to PA. When NHS-AA ester is used as a protein crosslinker, the ECM protein forms an amide bond directly with PA. (B) Outline of the PA gel preparation method. When sulfo-SANPAH is used as a protein crosslinker, $0.5 \mathrm{~mL}$ of sulfo-SANPAH solution ( $0.2 \mathrm{mg} / \mathrm{mL}$ ) was applied to the gel surface under $365 \mathrm{~nm}$ UV light after polymerization of AA and bis-AA (top). When NHS-AA ester is used as a protein crosslinker, it is mixed with $\mathrm{AA}$ and bis-AA before polymerization (bottom)

\section{INTRODUCTION}

Mechanical stimulation by the extracellular matrix (ECM) controls physiological and pathological cellular responses. In regenerative medicine, optimal stiffness of the culture substrate increases the efficiency of induction of differentiation and establishment of threedimensional (3D) culture systems for organ regeneration (Engler et al., 2006; Kolahi et al., 2012). In cancer biology, a relationship has been demonstrated between mammary tissue stiffness and breast cancer progression (Paszek and Weaver, 2004; Paszek et al., 2005; Levental et al., 2007, 2009; Butcher et al., 2009).

Various cell culture materials are used to investigate the effects of ECM stiffness on cellular responses, including biomaterials such as collagen (Parenteau-Bareil et al., 2010), Matrigel (Kleinman et al., 1986), polysaccharide (Baldwin and Kiick, 2010), polyacrylamide (PA) (Kandow et al., 2007), polyethylene glycol (PEG) (Zhu, 2010), and self-assembling peptides (Koutsopoulos, 2016). Among them, PA gels are most widely used to study cellECM mechanical interactions, because of their convenient usage, biocompatibility, and reproducibility of stiffness (Kandow et al., 2007; Tilghman et al., 2010; Dupont et al., 2011; Wen et al., 2014; Tsou et al., 2016; Domura et al., 2017; Martín et al., 2017).

PA gels require protein crosslinkers to crosslink ECM proteins to the gel for cell adhesion. Sulfosuccinimidyl 6 - $\left(4^{\prime}\right.$-azido- $2^{\prime}$ - nitrophenylamino)hexanoate (sulfo-SANPAH) is a commonly used PA gel crosslinker (Pelham and Wang, 1997). ECM proteins displace the sulfosuccinimidyl groups of sulfoSANPAH molecules, forming amide bonds (Figure 1A). However, ECM protein binding by sulfo-SANPAH is unstable and can result in inconsistent effects of gel stiffness on cellular responses (Kandow et al., 2007; Yip et al., 2013). This is presumably due to the nonspecific binding of sulfo-SANPAH to the PA gel through ultraviolet (UV) irradiation (Figure 1B, top). In addition, the low solubility and high cost of sulfo-SANPAH can hinder experiments that require large amounts of PA gel (Pelham and Wang, 1997; Kandow et al., 2007). To improve the stability of protein bound to PA gels, several alternative methods have been developed, which attach ECM proteins to the gels by covalent bonds using 1-ethyl-3-(3-dimethylaminopropyl)carbodiimide (Beningo and Wang, 2002; Kandow et al., 2007), N-acryloyl-6aminocaproic acid (ACA) (Yip et al., 2013), or the N-succinimidyl ester of acrylamidohexanoic acid (N6; Table 1) (Willcox et al., 2005; Kandow et al., 2007). However, these crosslinkers have not been widely accepted as sulfo-SANPAH alternatives because they require long reaction times or are commercially unavailable.

NHS has a long history of use as an easy-to-dissolve and economical protein crosslinker and is used in the ACA and N6 protocols to initiate substitution reactions with ECM proteins. 
TABLE 1 | List of PA gel protocol.

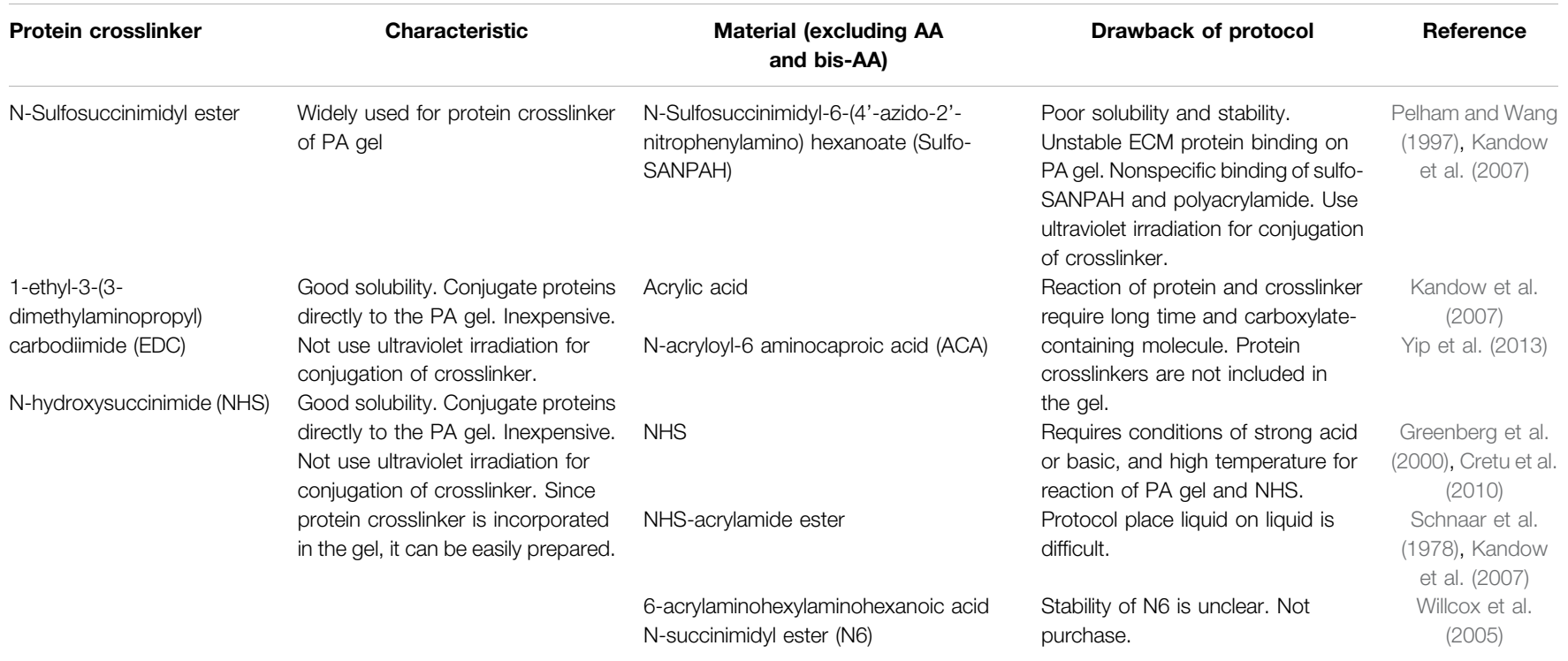

However, direct conjugation of NHS to acrylamide (AA) would simplify the protocol, as ECM proteins could be covalently bound directly to AA through a nucleophilic acyl substitution reaction (Figure 1A, bottom). Only one study has reported a protocol for the preparation method of PA gels using NHS, in which NHS was mixed with an AA/bis-AA mixture before polymerization (Cretu et al., 2010). However, this protocol is practically difficult, because NHS binding to AA requires esterification of the amide group, which only occurs under nonphysiological conditions involving strong acids or bases in high temperatures (Greenberg et al., 2000). NHS-AA ester is a commercially available alternative that avoids this problem. NHS-AA ester, dissolved in toluene, is applied to an AA/bis-AA mixture prior to polymerization (Schnaar and Lee, 1975; Schnaar et al., 1978; Kandow et al., 2007); however, it is difficult to uniformly distribute NHS on the gel surface by pouring liquid on liquid, and thus impractical. A practical method would require mixing NHS-AA ester with AA and bis-AA before polymerization (Figure 1B, bottom).

In this study, we developed a new preparation method for PA gels using NHS-AA ester, and demonstrated its utility in studying the effects of ECM stiffness on cellular behavior. We optimized the ratios of NHS-AA ester and AA to achieve similar stiffness to sulfo-SANPAH gels and accomplished uniform ECM protein coating efficacy on gels on various stiffness. In addition, we compared cellular behaviors on gels containing NHS-AA ester and sulfo-SANPAH. The results suggest that NHS-AA estercontaining PA gels can be used as an inexpensive and reproducible alternative to sulfo-SANPAH-containing gels.

\section{MATERIALS AND METHODS}

\section{Cell Culture}

MCF7 cells were obtained from the Health Science Research Resources Bank (Osaka, Japan). The cells were maintained in
Dulbecco's modified Eagle's medium (DMEM) containing 10\% fetal bovine serum, $100 \mathrm{U} / \mathrm{mL}$ penicillin, and $100 \mu \mathrm{g} / \mathrm{mL}$ streptomycin (Fujifilm Wako Pure Chemical, Osaka, Japan). MCF10A cells were obtained from American Type Culture Collection (Manassas, VA, United States), and maintained in DMEM/F12 (Nakarai Tesque Inc., Kyoto, Japan) supplemented with $20 \mathrm{ng} / \mathrm{mL}$ of epidermal growth factor (EGF) (Peptide Institute Inc., Osaka, Japan), $100 \mathrm{ng} / \mathrm{mL}$ of cholera toxin (Sigma, St. Louis, MO, United States), $0.01 \mathrm{mg} / \mathrm{mL}$ of insulin (Sigma), $500 \mathrm{ng} / \mathrm{mL}$ of hydrocortisone (Fujifilm Wako Pure Chemical), 5\% horse serum (Thermo Fisher Scientific, Waltham, MA, United States), $100 \mathrm{U} / \mathrm{mL}$ penicillin, and $100 \mu \mathrm{g} / \mathrm{mL}$ streptomycin (Debnath et al., 2003). All cells were maintained at $37^{\circ} \mathrm{C}$ in a humidified $5 \% \mathrm{CO}_{2} / 95 \%$ air atmosphere.

\section{Antibodies}

Anti-p-FAK antibodies (Tyr397; ab81298), Alexa Fluor ${ }^{\circledR} 488$ Donkey Anti-Rabbit IgG (ab150062), and Alexa Fluor ${ }^{\circledR} 488$ Goat Anti-Mouse IgG (ab150113) were purchased from Abcam (Cambridge, United Kingdom). Anti-YAP antibodies (sc-101199) were purchased from Santa Cruz Biotechnology, Inc. (Dallas, TX, United States).

\section{Preparation of Aminosilanated (Bottom) and Chlorosilanated (Top) Coverslips}

For the bottom coverslips, $1 \mathrm{~mL}$ of $0.1 \mathrm{M} \mathrm{NaOH}$ (Fujifilm Wako Pure Chemical) was applied to a $22 \mathrm{~mm}$ coverslip (Matsunami Glass Ind., Ltd., Osaka, Japan) for $3 \mathrm{~min}$ to increase their reactivity with aminosilane. The $\mathrm{NaOH}$ was aspirated, and the coverslips were dried. Next, $300 \mu \mathrm{L}$ of 3-aminopropyltriethoxysilane (APTES; Tokyo Chemical Industry Co., Ltd, Tokyo, Japan) was applied to the coverslip for $3 \mathrm{~min}$. Then, the solution was aspirated, and the coverslip was washed three times with deionized water (DW) for $10 \mathrm{~min}$ each, dried, and $500 \mu \mathrm{L}$ of $0.5 \%$ glutaraldehyde (Tokyo 
TABLE 2 | Recipe of polyacrylamide using NHS-AA ester and sulfo-SANPAH as protein crosslinker.

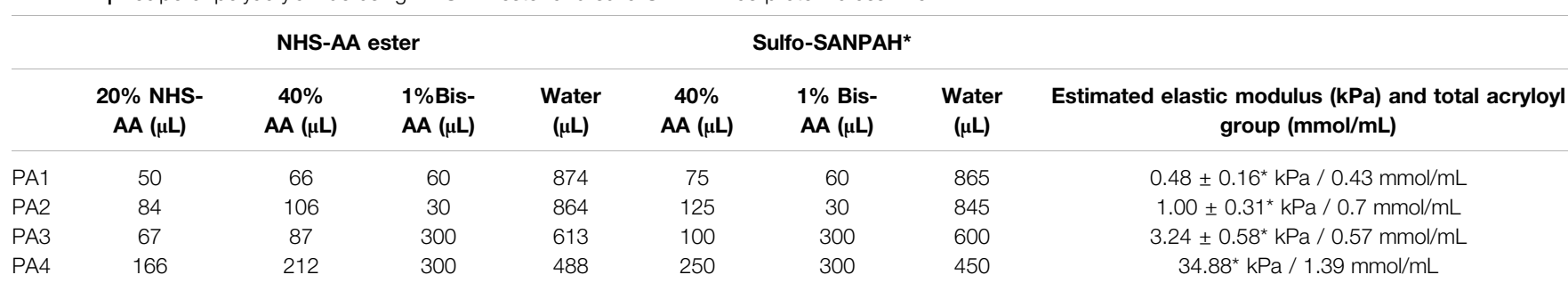

*We used method of Tse and Engler 2010 as reference (Tse and Engler, 2010).

Chemical Industry Co., Ltd) was applied for $30 \mathrm{~min}$ to activate the APTES. After incubation, the solution was aspirated, and the coverslip was washed three times with DW for $10 \mathrm{~min}$ each. The dried coverslips could be stored at room temperature (RT) for several days. For the top coverslips, $120 \mu \mathrm{L}$ of dichlorodimethylsilane (DCDMS) (Tokyo Chemical Industry Co., Ltd) was added to the coverslip and allowed to react for $5 \mathrm{~min}$. Then, the solution was aspirated and the coverslip was washed with DW for $1 \mathrm{~min}$.

\section{Preparation of PA Gel Containing NHS-AA Ester}

The amounts of NHS-AA ester and AA used were as previously described (Tse and Engler, 2010). We prepared the gel by mixing $40 \%$ AA (Fujifilm Wako Pure Chemical) and 1\% bis-AA (Bio-Rad Laboratories, Inc., Hercules, CA, United States) in Milli-Q water and 20\% NHS-AA ester (Tokyo Chemical Industry Co., Ltd.) in toluene (Table 2). The NHS-AA mixture was incubated for $5 \mathrm{~min}$ to transfer NHS-AA ester from the organic phase to the aqueous phase. After incubation, the solution was centrifuged for $5 \mathrm{~min}$ at $500 \mathrm{~g}$ and the toluene was removed. The soft gel solutions were degassed for $30 \mathrm{~min}$ in vacuum. To activate polymerization, $10 \mu \mathrm{L}$ of $10 \%$ ammonium persulfate (APS) and $1 \mu \mathrm{L}$ of tetramethylethylenediamine (TEMED) $(1 \%$ and $0.1 \%$ of total volume, respectively) (both from Fujifilm Wako Pure Chemical) were added and briefly mixed. Then, $25 \mu \mathrm{L}$ of the mixture was placed on a bottom coverslip and covered with a top coverslip. The sandwiched gels were incubated for $30 \mathrm{~min}$ (PA1), $20 \mathrm{~min}$ (PA2 and PA3), and $10 \mathrm{~min}$ (PA4) at RT, and then the top coverslips were removed. The polymerized gels were transferred to 6-well plates and washed three times for $5 \mathrm{~min}$ with $2 \mathrm{~mL}$ of phosphate-buffered saline (PBS) to remove unreacted AA. Gels were incubated with coating proteins overnight at $4^{\circ} \mathrm{C}$. Unreacted NHS in the wells was blocked with $0.1 \%$ bovine serum albumin (BSA, Fujifilm Wako Pure Chemical) in DMEM for $30 \mathrm{~min}$. Preparation process of PA gel containing NHS-AA ester is summarized as below.

(Preparation of bottom coverslips)

1. $1 \mathrm{~mL}$ of $0.1 \mathrm{M} \mathrm{NaOH}$ Is Applied to Coverslips for $3 \mathrm{~min}$.

2. The $\mathrm{NaOH}$ was aspirated, and the coverslips are dried.

3. Next, $300 \mu \mathrm{L}$ of APTES is applied to the coverslip for $3 \mathrm{~min}$, and then is aspirated.

4. The coverslip is washed three times with DW for $10 \mathrm{~min}$ each, and then is dried.

5. $500 \mu \mathrm{L}$ of $0.5 \%$ Glutaraldehyde Is Applied for $30 \mathrm{~min}$.
6. After incubation, the solution is aspirated, and the coverslip is washed three times with DW for $10 \mathrm{~min}$ each. (The dried coverslips can be stored at RT for several days.)

(Preparation of top coverslips)

7. $120 \mu \mathrm{L}$ of DCDMS Is Added to the Coverslip and Allowed to React for $5 \mathrm{~min}$.

8. Then, the solution was aspirated, and the coverslip is washed with DW for $1 \mathrm{~min}$.

(Gel Preparation)

9. Mixing solutions are prepared by mixing $40 \%$ AA and $1 \%$ bisAA in Milli-Q water and 20\% NHS-AA ester in toluene (Table 2).

10. The Solutions Are Incubated for $5 \mathrm{~min}$.

11. After incubation, the solutions are centrifuged for $5 \mathrm{~min}$ at $500 \mathrm{~g}$ and then the toluene is removed.

12. The Solutions Are Degassed for $30 \mathrm{~min}$ Under Vacuum Conditions.

13. To activate polymerization, $10 \mu \mathrm{L}$ of $10 \%$ APS and $1 \mu \mathrm{L}$ of TEMED are added and briefly mixed.

14. Then, $25 \mu \mathrm{L}$ of the mixture is placed on bottom coverslip and coved with a top coverslip.

15. The sandwiched gels are incubated for $30 \mathrm{~min}$ for PA1, 20 min for PA2 and PA3, and 10 min for PA4 at RT, and then the top coverslips are removed.

16. The polymerized gels are transferred to 6-well plates and washed three times for $5 \mathrm{~min}$ with $2 \mathrm{~mL}$ of PBS.

17. The Gels Are Incubated With Coating Proteins Overnight at $4^{\circ} \mathrm{C}$.

\section{Preparation of PA Gels Using Sulfo-SANPAH}

Gels containing sulfo-SANPAH were prepared to contain equivalent amounts of acryloyl groups in NHS-AA ester gels (Table 2). After polymerization of AA and bis-AA, $0.5 \mathrm{~mL}$ of $0.2 \mathrm{mg} / \mathrm{mL}$ sulfo-SANPAH (Thermo Fisher Scientific) was applied to the gel surface under $365 \mathrm{~nm}$ UV light at RT for $10 \mathrm{~min}$. After the binding reaction with sulfo-SANPAH, the gel was washed three times with $2 \mathrm{~mL}$ PBS. These gels were proteincoated similar to NHS-AA esters gels. 


\section{Measurement of PA Gel Stiffness by Atomic Force Microscopy (AFM)}

The Young's modulus of elasticity, which represents the stiffness of PA gels, was measured with AFM for each type of gel. The prepared PA gels were washed twice with $2 \mathrm{~mL}$ PBS and placed in a $35-\mathrm{mm}$ dish. To measure their actual stiffness, the gels were immersed in PBS and force was measured on a NanoWizard three NanoOptics atomic force microscope (JPK Instruments, Berlin, Germany). Young's modulus was calculated for each force curve using JPK DP Data Processing Software (JPK Instruments), which uses a Hertzian contact model.

\section{Cloning, Expression, and Purification of EGFP}

A cDNA encoding EGFP was amplified by polymerase chain reaction (PCR) (KOD FX Neo; TOYOBO Co., Ltd, Osaka, Japan) using primers containing the BamHI (forward, $5^{\prime}$-cgggatccATGG TGAGCAAGGGCGAGGAGCTG- ${ }^{\prime}$ ) and the EcoRI (reverse, $5^{\prime}$-cggaattcTTACTTGTACAGCTCGTCCATGCCGAGAGTG-

$3^{\prime}$ ) restriction sites of pGEX-6P-1 (GE Healthcare, Chicago, IL, United States). The vector and PCR products were digested with BamHI/EcoRI and ligated together using T4 DNA ligase (TAKARA BIO Inc., Shiga, Japan). After plasmid purification and sequencing, the pGEX-6P-EGFP expression vector was transformed into BL21 (DE3) competent Escherichia coli (TAKARA BIO Inc.). Colonies were inoculated in $2 \mathrm{~L}$ of Luria-Bertani broth for large-scale culture, and GST-EGFP expression was induced with $0.5 \mathrm{mM}$ isopropyl $\beta$-d-1thiogalactopyranoside for $4 \mathrm{~h}$. The bacterial pellet was resuspended in $20 \mathrm{~mL}$ of lysis buffer $(50 \mathrm{mM}$ Tris, $1 \%$ TritonX-100, $150 \mathrm{mM} \mathrm{NaCl}, 5 \mathrm{mM} \mathrm{MgCl}, 1 \mathrm{mM}$ DTT, pH 7.5) and disrupted by sonication. Then, the lysate was added to a $50 \%$ glutathione Sepharose (Nakarai Tesque Inc.) slurry in $4 \mathrm{~mL}$ PBS and incubated at $4^{\circ} \mathrm{C}$ for $1 \mathrm{~h}$, and then washed with PBS. To elute EGFP, the beads were mixed with $2 \mathrm{~mL}$ of elution buffer (50 mM Tris-HCl, $150 \mathrm{mM} \mathrm{NaCl}, 1 \mathrm{mM}$ EDTA, $1 \mathrm{mM}$ DTT, $\mathrm{pH}$ 7.5) containing $360 \mathrm{U}$ of PreScission protease (GE Healthcare) and incubated at $4^{\circ} \mathrm{C}$ for $5 \mathrm{~h}$. The supernatant was collected and EGFP concentration was measured using a BCA Protein Assay Kit (Thermo Fisher Scientific).

\section{Quantitation of EGFP and Rhodamine-Fibronectin Coating of PA Gels} EGFP and rhodamine-fibronectin (Cytoskeleton Inc., Denver, $\mathrm{CO}$, United States) were conjugated to gels overnight. The gels were then mounted with VECTASHIELD ${ }^{\circledast}$ Antifade Mounting Medium (Novus Biologicals, Littleton, CO, United States) and examined on an Eclipse Ti microscope (Nikon Instruments Inc., Melville, NY, United States) fitted with a Plan Fluor 10× objective lens (numerical aperture (NA) 0.3; Nikon Instruments Inc.). The fluorescent intensities of EGFP and rhodamine-fibronectin were quantified using ImageJ v. 2.0.0-rc-69/1.52p (National Institutes of Health, Bethesda, MD, United States). Fluorescent intensities were calculated as the difference between the average fluorescent intensity of the proteins in each field and the background fluorescent intensity. Fluorescence from five randomly selected fields was measured.

\section{Cell Proliferation and Adhesion Assays}

The gels were coated with $100 \mu \mathrm{g} / \mathrm{mL}$ collagen I (Corning Inc, Corning, NY, United States). For proliferation assays, $1.0 \times 10^{5}$ cells were added to each well and incubated at $37^{\circ} \mathrm{C}$ for 3 days. For cell adhesion assays, $5.0 \times 10^{5}$ cells were added to each well and incubated at $37^{\circ} \mathrm{C}$ for $1 \mathrm{~h}$. In both assays, the cells were counted using cell counting kit-8 (CCK-8, Dojindo Molecular Technologies, Inc., Kumamoto, Japan). Absorbance was measured at $450 \mathrm{~nm}$ using a Sunrise microplate reader (Tecan Japan Co. Ltd., Kawasaki, Japan).

\section{Cell Area and Circularity}

Cells were examined on an Eclipse Ti microscope fitted with a Plan Fluor 20× objective lens (NA 0.45; Nikon Instruments Inc.). After binary image processing, the cell area and circularity were measured using ImageJ. Five randomly selected fields were considered.

\section{Immunofluorescence}

MCF10A cells $\left(5 \times 10^{4}\right)$ were cultured on collagen-coated PA gels for $24 \mathrm{~h}$. The cells were fixed with $4 \%$ paraformaldehyde for $20 \mathrm{~min}$ and permeabilized with $1 \%$ (for $p$-FAK) and $0.1 \%$ (for YAP) Triton X-100 in PBS for 10 min, and then blocked with 3\% BSA in PBS for $30 \mathrm{~min}$ at RT. The cells were incubated with 1:50 solution of primary antibodies, for $p$-FAK and YAP, overnight at $4^{\circ} \mathrm{C}$, and then incubated with secondary antibodies and Hoechst 33,342 (Thermo Fisher Scientific) at RT for $30 \mathrm{~min}$. Finally, the cells were mounted with VECTASHIELD ${ }^{\circledR}$ and examined on an Eclipse Ti microscope fitted with a Plan Fluor 20x objective lens (NA 0.45; Nikon Instruments Inc.).

\section{Matrigel Overlay Culture on PA Gel}

Matrigel overlay culture on PA gel was performed as previously described, with slight modifications (Debnath et al., 2003). MCF10A cells were detached with trypsin at $37^{\circ} \mathrm{C}$ for $5 \mathrm{~min}$ to ensure complete collection of the cells, and then resuspended in culture medium (DMEM/F12 supplemented with $20 \mathrm{ng} / \mathrm{mL}$ of EGF, $100 \mathrm{ng} / \mathrm{mL}$ of cholera toxin, $0.01 \mathrm{mg} / \mathrm{mL}$ of insulin, $500 \mathrm{ng} /$ $\mathrm{mL}$ of hydrocortisone, $5 \%$ horse serum, $100 \mathrm{U} / \mathrm{mL}$ penicillin, and $100 \mu \mathrm{g} / \mathrm{mL}$ streptomycin). The cells were washed with assay medium (DMEM/F12 supplemented with $100 \mathrm{ng} / \mathrm{mL}$ of cholera toxin, $0.01 \mathrm{mg} / \mathrm{mL}$ of insulin, $500 \mathrm{ng} / \mathrm{mL}$ of hydrocortisone, $2 \%$ horse serum, $100 \mathrm{U} / \mathrm{mL}$ penicillin and $100 \mu \mathrm{g} / \mathrm{mL}$ streptomycin), and then counted and diluted to $1 \times 10^{4}$ cells $/ \mathrm{mL}$ in assay medium at RT. We prepared $4 \%$ Matrigel solution in assay medium at $4^{\circ} \mathrm{C}$. Then, the cells and Matrigel solution were combined in 1:1 ratio. PA gels were transferred to 12 -well plates, washed with assay medium three times, and $1 \mathrm{~mL}$ of the cell-Matrigel mixture $\left(5 \times 10^{3}\right.$ cells/well $)$ was applied to the PA gel. The cells were refed assay medium containing 2\% Matrigel every 4 days. After 10 days, the cells were examined on an Eclipse Ti microscope fitted with a Plan Fluor $20 \times$ objective lens (NA 0.45; Nikon Instruments Inc.). 
A
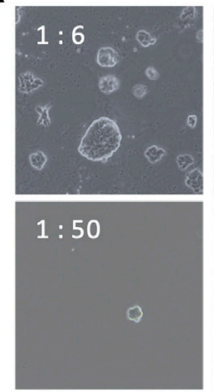

C

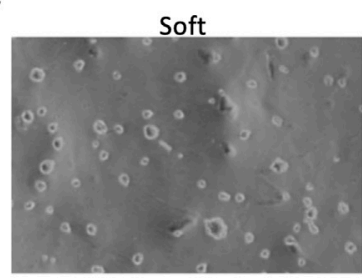

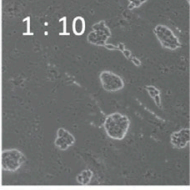

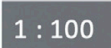

B

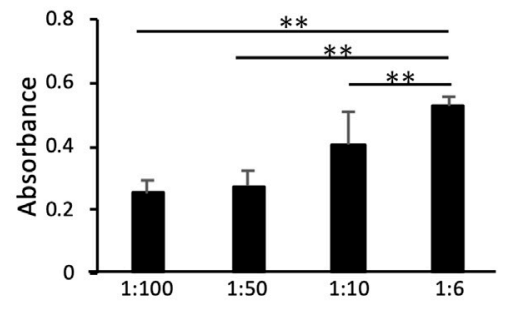

FIGURE 2 | Effects of NHS-AA ester:AA mixing ratios on cell proliferation and adhesion. Cell distribution and proliferation increased with a higher mixing ratio of NHS-AA ester:AA (A) Cells adhered to softer gels (estimated stiffness: $0.48 \mathrm{kPa}$ ) made with different NHS-AA ester:AA mixing ratios. Scale bar: 100 mm (B) Cell proliferation on gels made with different NHS-AA ester:AA mixing ratios. (C) The distribution of adherent cells was uniform on both soft and stiff gels (estimated stiffness: 0.48 and $34.88 \mathrm{kPa}$, respectively) at a mixing ratio of 1:6. Scale bar: $500 \mu \mathrm{m}$. (D) Cell proliferation rate was higher on stiffer gels than on softer ones with 1:6 mixing ratios. Each value represents the mean of three independent replicates \pm the $\mathrm{SD} .{ }^{\star *} p<0.01,{ }^{*} p<0.05$.

\section{Statistical Analysis}

Differences between pairs of groups were analyzed by two-tailed unpaired Student's $t$-test. $p$ values $<0.05$ were considered statistically significant. Error bars in all figures represent the mean of three independent experiments \pm the standard deviation (SD). Data for in vitro experiments represent the results of three independent biological replicates.

\section{RESULTS}

\section{Determination of the Optimal NHS-AA ester: AA Mixing Ratio}

In the development of a new PA gel preparation method, we aimed to create gels that simulate the stiffness of various tissues. We used previously described AA:bis-AA mixing ratios of PA gels using sulfo-SANPAH that resulted in four different stiffness levels $(0.48,1,3.24$, and $34.88 \mathrm{kPa}$ ) (Tse and Engler, 2010). We hypothesized that if we use the published amount of bis-AA and ensure that the total amount of acryloyl groups in the gel came from NHS-AA ester and AA together, the stiffness of the NHS-AA ester gels would stay the same as the reference gels, as NHS would be hydrolyzed and dissolved in water. According to this hypothesis, we determined the optimal NHS-AA ester:AA ratio at the four stiffness levels mentioned above.

With the optimal mixing ratio, the ECM proteins should uniformly coat gels of any stiffness when equal amounts of ECM proteins are applied. Excess surface crosslinker permits the saturated binding of a constant amount of ECM protein, and the amount of protein coating the surface is proportional to the amount of applied ECM protein (Beer et al., 2015). Therefore, we aimed to identify a mixing ratio that provided an excess of surface NHS and used it to confirm uniform ECM protein coating of different stiffness gels.

First, we optimized the highest NHS-AA ester:AA ratio that solidified the gel, since NHS-AA ester was presumed to inhibit the polymerization of AA and bis-AA. At a 1:5 ratio, the gel did not adequately solidify (data not shown), whereas polymerization was successful at ratios of 1:6 and lower. We examined the adhesion and proliferation of MCF7 cells on collagen I-coated gels of the softest stiffness (Table 2) at various mixing ratios. Adherent cells were evenly distributed on the gel at a mixing ratio of 1:6. The distribution of adherent cells became uneven (Figure 2A) and the proliferation significantly decreased (Figure 2B) as the proportion of NHS-AA ester decreased. Next, we examined the uniformity of cell adhesion on gels of different stiffness (PA1-4, with PA1 being the softest and PA4 being the stiffest; Table 2) at the mixing ratio of 1:6. Adhesion was uniform on gels of all stiffness levels (Figure 2C), while proliferation was significantly reduced on soft gels compared to stiff gels (Figure 2D). These results suggest that a mixing ratio of 1:6 enables adequate gel solidification and uniform ECM protein coating of gels of various stiffness.

\section{Elastic Moduli of PA Gels Made With NHS-AA Ester}

We measured the elastic moduli of 1:6 gels of four different stiffness levels (Table 2) by AFM and compared them with 


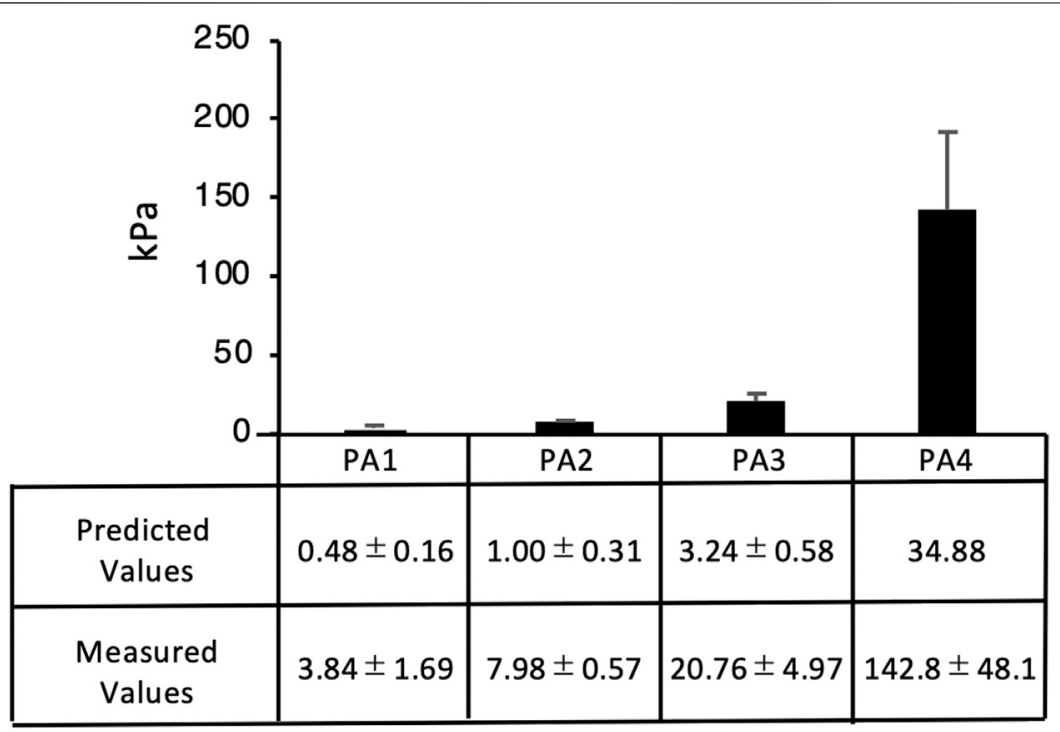

FIGURE 3| Elastic moduli of PA gels. Average elastic modulus values were measured by AFM. The elastic modulus of the four gels (PA1-4) was 3.84, 7.98, 20.76, and $142.8 \mathrm{kPa}$, respectively. Each value represents the mean of three independent replicates \pm the SD.

reference values (Tse and Engler, 2010). The elastic modulus values of each gel $(3.84,7.98,20.76$, and $142.8 \mathrm{kPa})$ were approximately three times higher than the estimated values $(0.48,1,3.24$ and $34.88 \mathrm{kPa})$, but the rates of changes in stiffness between the gels were equivalent to the estimated values (Figure 3).

\section{Confirmation of Protein Coating Efficacy on PA Gels Containing NHS-AA Ester}

We examined whether surface NHS was in sufficient excess for uniform ECM protein coating of gels at each stiffness level. The fluorescent intensity of enhanced green fluorescent protein (EGFP) reached saturation on both, the softest and the stiffest gels (PA1 and PA4, respectively) at mixing ratios above $1: 8$, indicating that excess NHS was present on the gel surface at these ratios (Figure 4A). There were no differences in EGFP coating efficacy among the gels of different stiffness, and the amount of EGFP coating the gels was proportional to the amount applied (Figure 4B). Equivalent ECM protein coating efficacy among gels of different stiffness was also confirmed using rhodamine-fibronectin (Figure 4C). Since the 1:6 mixing ratio was the maximum ratio that displayed equal protein coating efficacy at different stiffness levels, it was used in subsequent experiments.

\section{Comparison of Cell Behavior on PA Gels Containing NHS-AA Ester and Sulfo-SANPAH}

To confirm that the NHS-AA ester-containing PA gels could reproduce the results of previous biological experiments conducted with PA gels containing sulfo-SANPAH, we first compared cell adhesion, proliferation, and morphology on gels made with NHS-AA ester and sulfo-SANPAH. There were no significant differences in the adhesion of MCF7 and MCF10A cells on NHS-AA ester and sulfo-SANPAH gels of any stiffness (Figure 5A). However, both cell lines displayed increased proliferation on NHS-AA ester gels than on sulfoSANPAH gels (Figure 5B). With both crosslinkers, proliferation increased with increase in gel stiffness, while adhesion was unaffected by stiffness (Figures 5A,B). The morphology of MCF7 cells changed from circular to spreading with increasing stiffness, and similar changes observed on gels with either of the crosslinkers (Figure 5C). The cell area (Figure 5D) and circularity (Figure 5E) were similar in both cell lines on both gel types. These results indicate that gels containing NHS-AA ester induce cellular responses comparable to those containing sulfo-SANPAH.

\section{Immunostaining and Matrigel Overlay Culture on PA Gels Containing NHS-AA Ester}

To evaluate cellular responses to stiffness changes, we immunostained cells adhered to NHS-AA ester-containing PA gels for phosphorylated focal adhesion kinase ( $p$-FAK) and Yes1 associated transcriptional regulator (YAP). In MCF10A cells, focal adhesions stained with $p$-FAK increased in both size and number on stiff gels than on soft gels (Figure 6A). YAP localized to the cytoplasm of MCF10A cells adhered to soft gels but was translocated to the nucleus in cells on stiff gels (Figure 6B). Furthermore, in Matrigel overlay culture, MCF10A cells formed acini on soft gels and displayed a spread morphology on the surface of stiff gels (Figure 6C). These results indicate that PA gels using NHS-AA ester can reproduce the typical biological behaviors of cells on PA gels using sulfo-SANPAH. 
A

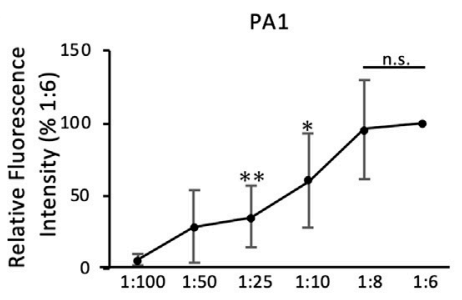

B

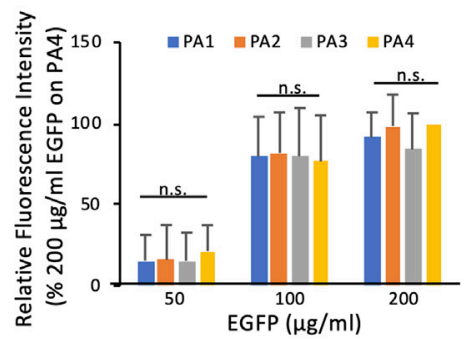

C

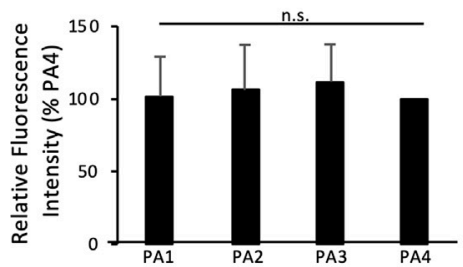

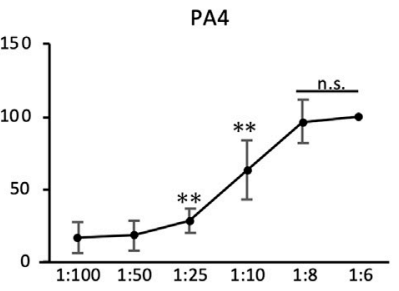

EGFP+

EGFP-

(Negative control)
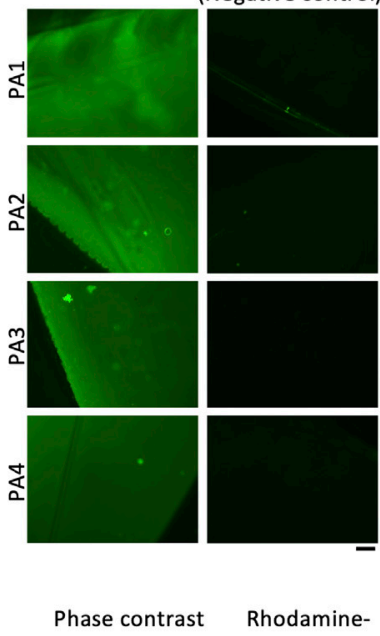

Rhodamine-

Fibronectin
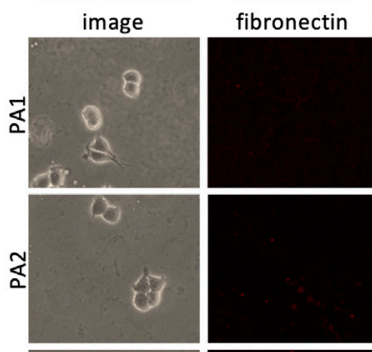

(Negative control)
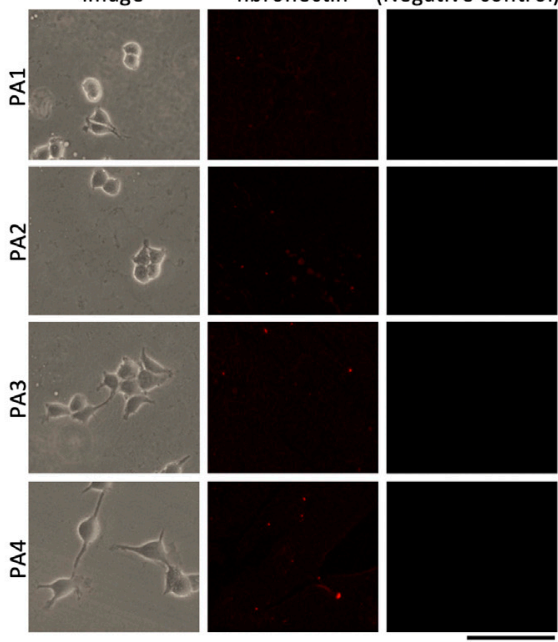

FIGURE 4 | Protein coating efficacy of PA gels containing NHS-AA ester. Protein coating efficacy was determined using EGFP and rhodamine-fibronectin (A) The fluorescent intensity of EGFP $(200 \mu \mathrm{g} / \mathrm{mL})$ reached saturation on both soft and stiff gels (PA1 and PA4, respectively) at mixing ratios above 1:8 (B) The amount of EGFP coating the gels was proportional to the amount applied (left), and EGFP coating efficacy was similar among gels of different stiffness (right) (C) Rhodamine-fibronectin coating was similar among gels of different stiffness. The mean of three independent replicates \pm the SD (left) are shown. Scale bar: $100 \mu$ m. ${ }^{\star \star} p<0.01$, ${ }^{\star} p<0.05$, n.s., not significant.

\section{DISCUSSION}

In this study, we have developed a new preparation method for PA gels using NHS-AA ester. These gels overcome the disadvantages of sulfo-SANPAH and can be produced for less than $1 \%$ the cost of sulfo-SANPAH gels using a simpler preparation procedure than other alternative methods. Due to the excess surface NHS and the covalent nature of the bonds formed, the ECM protein on gels containing NHS-AA ester is uniform and stable, and efficiently transmits the stiffness of gels to cells. Importantly, cellular responses to changes in gel stiffness efficiently replicated those reported for sulfo-SANPAH gels. 
A

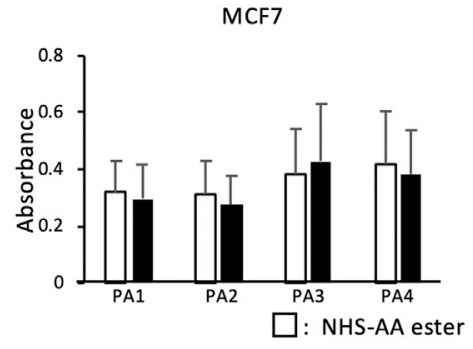

B

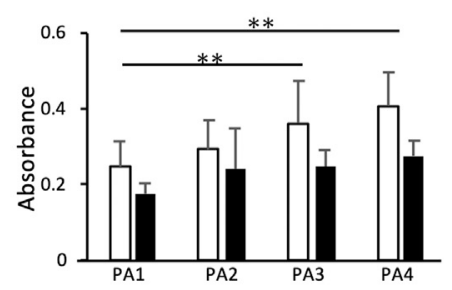

C

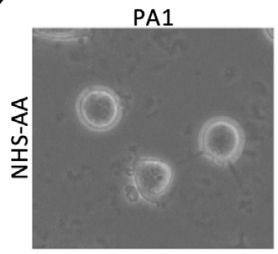

$\square$ : NHS-AA ester

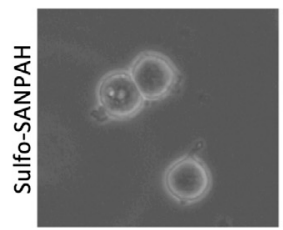

D

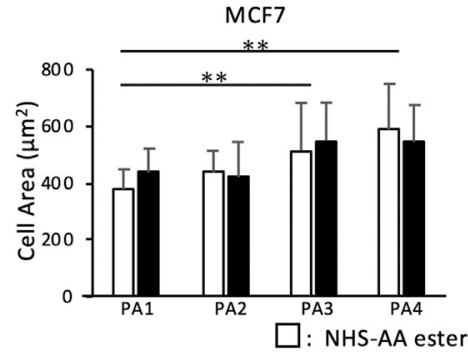

E

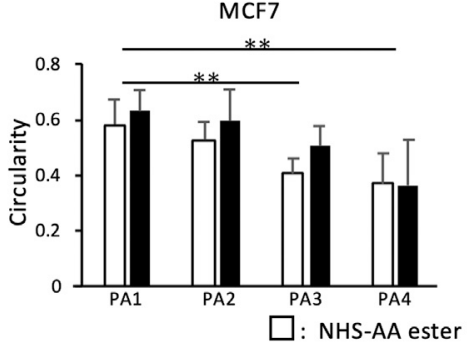

MCF10A

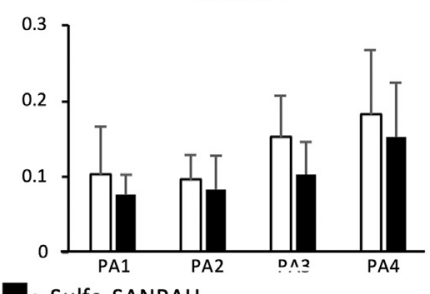

: Sulfo-SANPAH

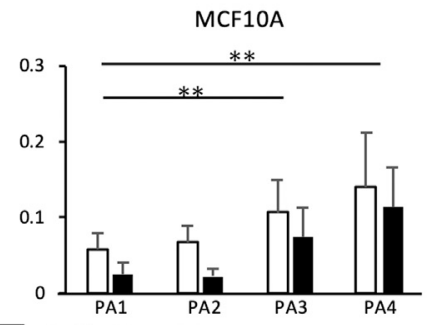

: Sulfo-SANPAH
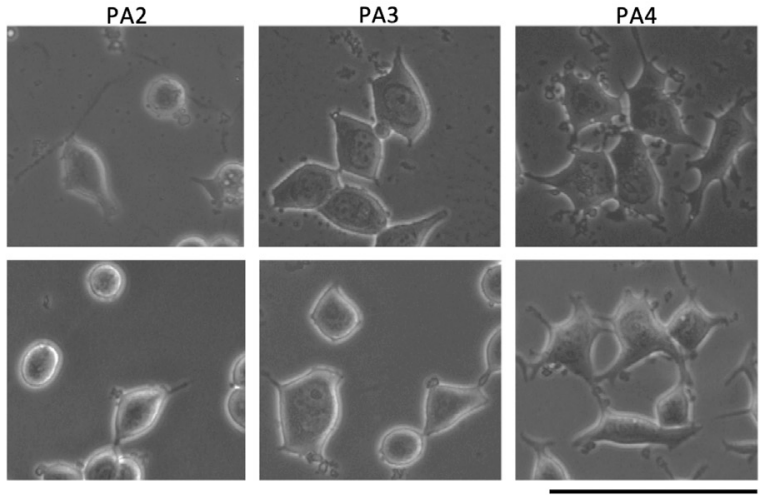

MCF10A

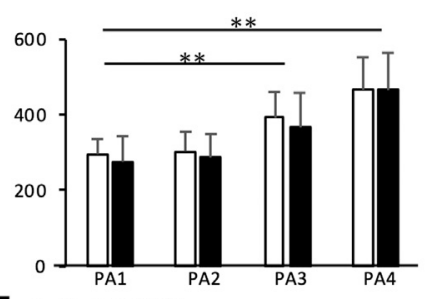

: Sulfo-SANPAH

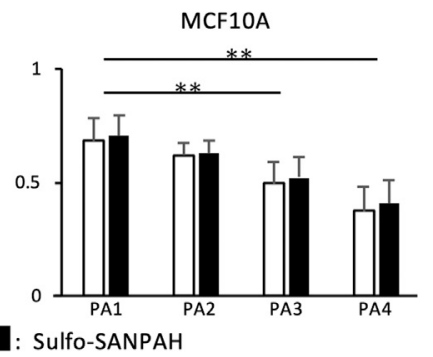

FIGURE 5 | Comparison of cell adhesion, proliferation, and morphology on PA gels containing NHS-AA ester and sulfo-SANPAH. (A) Cell adhesion of MCF7 (left) and MCF10A (right) cells showed no difference on gels of different stiffness containing NHS-AA ester (white) and sulfo-SANPAH (black) (B) The proliferation rate of MCF7 (left) and MCF10A (right) cells was higher on NHS-AA ester gels (white) than on sulfo-SANPAH gels (black), irrespective of stiffness. The proliferation increased on stiffer gels (C) No difference in MCF7 cell morphology was observed between the two gels at any stiffness. Scale bar: 100 um. (D-E) Cell area (D) and circularity (E) of MCF7 (left) and MCF10A (right) cells showed no difference on gels of different stiffness containing NHS-AA ester (white) and sulfo-SANPAH (black). The morphology of both cells changed from circular to spreading with increased stiffness. Values represent the mean of three independent replicates \pm the SD. ${ }^{\star \star} p<0.01$, ${ }^{*} p<0.05$. 


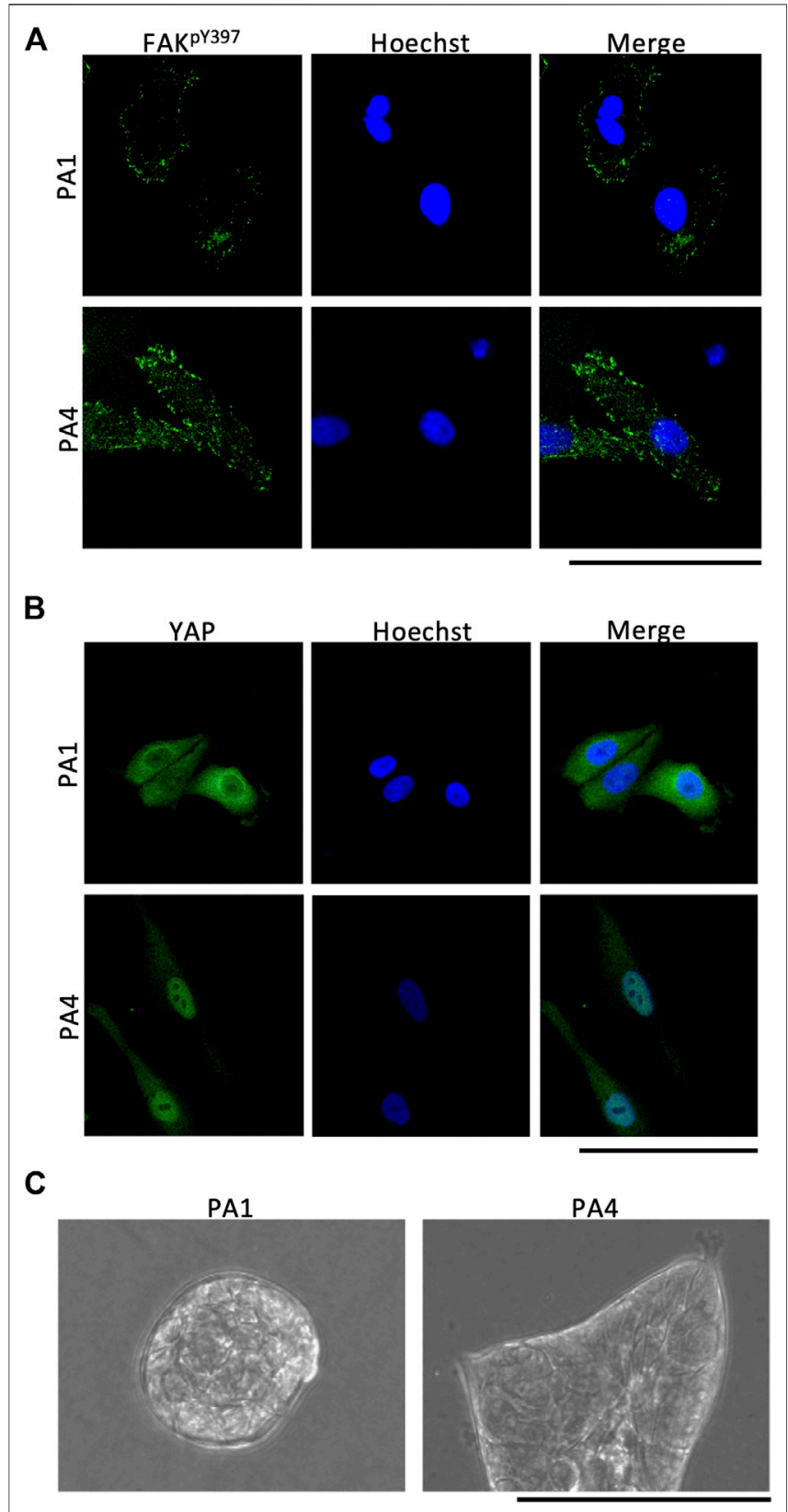

FIGURE 6 | Immunostaining and Matrigel overlay culture of PA gels containing NHS-AA ester. (A) Focal adhesions stained with $p$-FAK increased more in size and number on stiff gels (bottom) than on soft gels (top). Scale bar: $100 \mu \mathrm{m}$ (B) YAP protein in MCF10A cells was localized in the cytoplasm on soft gels (top) and in the nucleus on stiff gels (bottom). Scale bar: $100 \mu \mathrm{m}$. (C) In Matrigel overlay culture, MCF10A cells formed acini on soft gels and spread on stiff gels.

With the goal of a simple preparation method, we mixed NHS-AA ester with AA and bis-AA before polymerization. When compared with gels containing sulfo-SANPAH or other alternative protein crosslinkers like ACA or N6, the preparation of PA gels containing NHS-AA ester is simpler. Since it is difficult to have equal number of crosslinking sites on gels of every stiffness, we provided an excess of surface NHS to ensure equivalent ECM protein coating. Therefore, the amounts of NHS contained in gels of each stiffness may be nonuniform, but the excess NHS enables ligand saturation, resulting in uniform ECM protein coating at all stiffness levels. Mixing NHS-AA ester into the gel results in NHS hydrolysis, resulting in negatively charged carboxyl groups that could impair gel durability (Kandow et al., 2007; Lee et al., 2009). However, in our experience, the durability of the gels containing NHS-AA ester was not noticeably different from that of gels containing sulfo-SANPAH.

We estimated that the gel stiffness levels established for sulfoSANPAH-containing gels would be maintained in NHS-AA estercontaining gels if equal amounts of acryloyl groups were incorporated. However, the elastic modulus values of NHS-AA ester-containing PA gels of respective stiffness were approximately three times the reference values of gels containing sulfo-SANPAH. Nonetheless, the rates of increases in stiffness among the gels were equivalent to the estimated values (Table 2) and cellular responses on gels with NHS-AA ester were similar to those with sulfo-SANPAH. This suggests that the observed differences between the measured and estimated values may be due to differences in the AFM systems used.

Despite their advantages, NHS-AA ester-containing PA gels do have some limitations. ECM proteins must be coated immediately after polymerization, because NHS-AA ester is quickly hydrolyzed, whereas gels for sulfo-SANPAH experiments can be stored for approximately 3 days prior to sulfo-SANPAH conjugation before the stiffness changes (Denisin and Pruitt, 2016). In this study, we used glass coverslips with a diameter of $22 \mathrm{~mm}$, as we found that the surface tension of the acrylamide mixture hindered the generation of larger or smaller gels by the described sandwich method. However, a modified preparation method for smaller scale gels used in drug screening studies has been reported (Medina et al., 2019). To investigate the effects of ECM stiffness on cellular responses, several 3D culture systems have recently been developed (Chaudhuri et al., 2014; Neves et al., 2020; Yamada et al., 2020). Although 3D culture systems are close to the biological environment, they are unable to separate the effects of structure and stiffness on cellular behavior. Thus, the PA gel culture will continue to be a useful experimental system for analyzing the effects of stiffness on cellular behavior.

In this study, we have developed a novel preparation method for PA gels using NHS-AA ester as a protein crosslinker. ECM proteins covalently bind to PA on these gels which efficiently transmit the stiffness of gels to cells. These economical gels are simple to make, highly reproducible, and allow experiments with large number of PA gels. The PA gels using NHS-AA ester is a practical and inexpensive alternative to sulfo-SANPAH gels in the evaluation of cell-ECM mechanical interactions. They may also contribute to advancements in various research areas, such as cancer biology, regenerative medicine, and embryology.

\section{DATA AVAILABILITY STATEMENT}

The original contributions presented in the study are included in the article/Supplementary Material, further inquiries can be directed to the corresponding author. 


\section{AUTHOR CONTRIBUTIONS}

YY designed the experiments; JK and YY performed the experiments, analyzed data, and wrote the manuscript; $\mathrm{MH}$ measured elastic moduli of PA gels with AFM; SS cloned EGFP; all authors reviewed the manuscript.

\section{FUNDING}

This work was supported by JSPS KAKENHI (Grant No. 19K18482 and 18K16641). This funding supported our study design, data collection, analysis and interpretation, and writing the manuscript. This work was also supported by JSPS KAKENHI (Grant No. 18K14063) and the Nanotech

\section{REFERENCES}

Baldwin, A. D., and Kiick, K. L. (2010). Polysaccharide-modified synthetic polymeric biomaterials. Biopolymers. 94, 128-140. doi:10.1002/bip.21334

Beer, M. V., Hahn, K., Diederichs, S., Fabry, M., Singh, S., Spencer, S. J., et al. (2015). Quantifying ligand-cell interactions and determination of the surface concentrations of ligands on hydrogel films: the measurement challenge. Biointerphases, 10, 021007. doi:10.1116/1.4919015

Beningo, K. A., and Wang, Y. (2002). Fc-receptor-mediated phagocytosis is regulated by mechanical properties of the target. J. Cell Sci. 115, 849-856.

Butcher, D. T., Alliston, T., and Weaver, V. M. (2009). A tense situation: forcing tumour progression. Nat. Rev. Cancer. 9, 108-122. doi:10.1038/nrc2544

Chaudhuri, O., Koshy, S. T., Branco Da Cunha, C., Shin, J. W., Verbeke, C. S., Allison, K. H., et al. (2014). Extracellular matrix stiffness and composition jointly regulate the induction of malignant phenotypes in mammary epithelium. Nat. Mater. 13, 970-978. doi:10.1038/nmat4009

Cretu, A., Castagnino, P., and Assoian, R. (2010). Studying the effects of matrix stiffness on cellular function using acrylamide-based hydrogels. J. Vis. Exp. 10 (42), 2089. doi:10.3791/2089

Debnath, J., Muthuswamy, S. K., and Brugge, J. S. (2003). Morphogenesis and oncogenesis of MCF-10A mammary epithelial acini grown in threedimensional basement membrane cultures. Methods. 30, 256-268. doi:10. 1016/S1046-2023(03)00032-X

Denisin, A. K., and Pruitt, B. L. (2016). Tuning the range of polyacrylamide gel stiffness for mechanobiology applications. ACS Appl. Mater. Interfaces. 8, 21893-21902. doi:10.1021/acsami.5b09344

Domura, R., Sasaki, R., Ishikawa, Y., and Okamoto, M. (2017). Cellular morphology-mediated proliferation and drug sensitivity of breast cancer cells. J. Funct. Biomater. 8, 18. doi:10.3390/jfb8020018

Dupont, S., Morsut, L., Aragona, M., Enzo, E., Giulitti, S., Cordenonsi, M., et al. (2011). Role of YAP/TAZ in mechanotransduction. Nature. 474, 179-183. doi:10.1038/nature10137

Engler, A. J., Sen, S., Sweeney, H. L., and Discher, D. E. (2006). Matrix elasticity directs stem cell lineage specification. Cell. 126, 677-689. doi:10.1016/j.cell. 2006.06.044

A. Greenberg, C. M. Breneman, and J. F. Liebman (Editors) (2000). The amide linkage: structural significance in chemistry, biochemistry, and materials science. Hoboken, NJ: John Wiley and Sons.

Kandow, C. E., Georges, P. C., Janmey, P. A., and Beningo, K. A. (2007). Polyacrylamide hydrogels for cell mechanics: steps toward optimization and alternative uses. Methods Cell Biol. 83, 29-46. doi:10.1016/S0091-679X(07) 83002-0

Kleinman, H. K., Mcgarvey, M. L., Hassell, J. R., Star, V. L., Cannon, F. B., Laurie, G. W., et al. (1986). Basement membrane complexes with biological activity. Biochemistry. 25, 312-318. doi:10.1021/bi00350a005

Kolahi, K. S., Donjacour, A., Liu, X., Lin, W., Simbulan, R. K., Bloise, E., et al. (2012). Effect of substrate stiffness on early mouse embryo development. PloS One. 7, e41717. doi:10.1371/journal.pone.0041717
Career-up Alliance and the Kyoto University Nano Technology Hub "Nanotechnology Platform Project" sponsored by the Ministry of Education, Culture, Sports, Science and Technology, Japan. It also had an important role in AFM measurement.

\section{ACKNOWLEDGMENTS}

The authors are grateful to Hidemitsu Nakagawa, Tomoyuki Yamaguchi, Toshie Shinagawa, and Masashi Kishi for critical discussion. We would like to thank Nozaki Tokushukai Hospital for financial support and Editage (www.editage.com) for English language editing.

Koutsopoulos, S. (2016). Self-assembling peptide nanofiber hydrogels in tissue engineering and regenerative medicine: progress, design guidelines, and applications. J. Biomed. Mater. Res. 104, 1002-1016. doi:10.1002/jbm.a.35638

Lee, H., Rho, J., and Messersmith, P. B. (2009). Facile conjugation of biomolecules onto surfaces via mussel adhesive protein inspired coatings. Adv. Mater. 21, 431-434. doi:10.1002/adma.200801222

Levental, I., Georges, P. C., and Janmey, P. A. (2007). Soft biological materials and their impact on cell function. Soft Matter. 3, 299-306. doi:10.1039/b610522j

Levental, K. R., Yu, H., Kass, L., Lakins, J. N., Egeblad, M., Erler, J. T., et al. (2009). Matrix crosslinking forces tumor progression by enhancing integrin signaling. Cell. 139, 891-906. doi:10.1016/j.cell.2009.10.027

Martín, C., Merino, S., González-Domínguez, J. M., Rauti, R., Ballerini, L., Prato, M., et al. (2017). Graphene improves the biocompatibility of polyacrylamide hydrogels: 3D polymeric scaffolds for neuronal growth. Sci. Rep. 7. doi:10.1038/s41598-017-11359-x

Medina, S. H., Bush, B., Cam, M., Sevcik, E., Delrio, F. W., Nandy, K., et al. (2019). Identification of a mechanogenetic link between substrate stiffness and chemotherapeutic response in breast cancer. Biomaterials, 202, 1-11. doi:10. 1016/j.biomaterials.2019.02.018

Neves, M. I., Moroni, L., and Barrias, C. C. (2020). Modulating alginate hydrogels for improved biological performance as cellular 3D microenvironments. Front. Bioeng. Biotechnol. 8, 665. doi:10.3389/fbioe.2020.00665

Parenteau-Bareil, R., Gauvin, R., and Berthod, F. (2010). Collagen-Based biomaterials for tissue engineering applications. Materials. 3, 1863-1887. doi: $10.3390 / \mathrm{ma} 3031863$

Paszek, M. J., and Weaver, V. M. (2004). The tension mounts: mechanics meets morphogenesis and malignancy. J. Mammary Gland Biol. Neoplasia. 9, 325-342. doi:10.1007/s10911-004-1404-x

Paszek, M. J., Zahir, N., Johnson, K. R., Lakins, J. N., Rozenberg, G. I., Gefen, A., et al. (2005). Tensional homeostasis and the malignant phenotype. Cancer Cell. 8, 241-254. doi:10.1016/j.ccr.2005.08.010

Pelham, R. J., and Wang, Y. (1997). Cell locomotion and focal adhesions are regulated by substrate flexibility. Proc. Natl. Acad. Sci. U.S.A. 94, 13661-13665. doi:10.1073/pnas.94.25.13661

Schnaar, R. L., and Lee, Y. C. (1975). Polyacrylamide gels copolymerized with active esters. A new medium for affinity systems Biochemistry. 14, 1535-1541. doi:10.1021/bi00678a030

Schnaar, R. L., Weigel, P. H., Kuhlenschmidt, M. S., Lee, Y. C., and Roseman, S. 1978). Adhesion of chicken hepatocytes to polyacrylamide gels derivatized with N-acetylglucosamine. J. Biol. Chem. 253, 7940-7951. doi:10.1016/S00219258(17)34462-9

Tilghman, R. W., Cowan, C. R., Mih, J. D., Koryakina, Y., Gioeli, D., Slack-davis, J. K., et al. (2010). Matrix rigidity regulates cancer cell growth and cellular phenotype. PloS One. 5, e12905. doi:10.1371/journal.pone.0012905

Tse, J. R., and Engler, A. J. (2010). Preparation of hydrogel substrates with tunable mechanical properties. Curr. Protoc. Cell Biol. Chapter 10, Unit 10.16. doi:10. 1002/0471143030.cb1016s47

Tsou, Y. H., Khoneisser, J., Huang, P. C., and Xu, X. (2016). Hydrogel as a bioactive material to regulate stem cell fate. Bioact. Mater. 1, 39-55. doi:10.1016/j. bioactmat.2016.05.001 
Wen, J. H., Vincent, L. G., Fuhrmann, A., Choi, Y. S., Hribar, K. C., Taylor-weiner, $\mathrm{H}$., et al. (2014). Interplay of matrix stiffness and protein tethering in stem cell differentiation. Nat. Mater. 13, 979-987. doi:10.1038/NMAT4051

Willcox, P. J., Reinhart-king, C. A., Lahr, S. J., Degrado, W. F., and Hammer, D. A. (2005). Dynamic heterodimer-functionalized surfaces for endothelial cell adhesion. Biomaterials. 26, 4757-4766. doi:10.1016/j.biomaterials.2004. 11.060

Yamada, Y., Yoshida, C., Hamada, K., Kikkawa, Y., and Nomizu, M. (2020). Development of three-dimensional cell culture scaffolds using laminin peptideconjugated agarose microgels. Biomacromolecules. 21, 3765-3771. doi:10.1021/ acs.biomac.0c00871

Yip, A. K., Iwasaki, K., Ursekar, C., MacHiyama, H., Saxena, M., Chen, H., et al. (2013). Cellular response to substrate rigidity is governed by either stress or strain. Biophys. J. 104, 19-29. doi:10.1016/j.bpj.2012.11.3805
Zhu, J. (2010). Bioactive modification of poly(ethylene glycol) hydrogels for tissue engineering. Biomaterials. 31, 4639-4656. doi:10.1016/j.biomaterials.2010.02.044

Conflict of Interest: The authors declare that the research was conducted in the absence of any commercial or financial relationships that could be construed as a potential conflict of interest.

Copyright $\odot 2021$ Kumai, Sasagawa, Horie and Yui. This is an open-access article distributed under the terms of the Creative Commons Attribution License (CC BY). The use, distribution or reproduction in other forums is permitted, provided the original author(s) and the copyright owner(s) are credited and that the original publication in this journal is cited, in accordance with accepted academic practice. No use, distribution or reproduction is permitted which does not comply with these terms. 